\title{
Correction to: Simulated microgravity significantly altered metabolism in epidermal stem cells
}

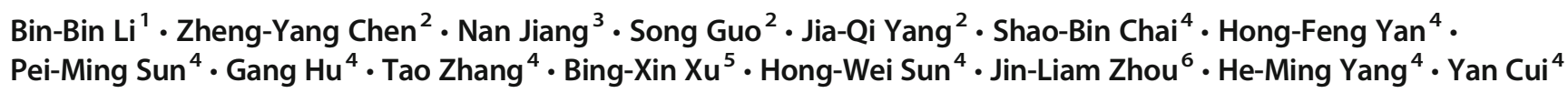

Published online: 23 June 2020

(C) The Society for In Vitro Biology 2020

\section{Correction to: In Vitro Cellular \& Developmental Biology - Animal 56, 200-212(2020)} https://doi.org/10.1007/s11626-020-00435-8

The affiliation given for Yan Cui in this article is not correct. The following is the correction affiliation: Department of General Surgery, PLA 306 Clinical Hospital of Anhui Medical University, Beijing 100101, China Also, the postal code provided for Bin-Bin Li needs correction. The correct postal code is: Beijing 100101
The online version of the original article can be found at https://doi.org/ $10.1007 / \mathrm{s} 11626-020-00435-8$

\author{
Pei-Ming Sun \\ 155363294@qq.com \\ Gang Hu \\ 916787543@qq.com \\ Tao Zhang \\ zt_102@163.com \\ Bing-Xin Xu \\ xubingxin75@163.com \\ Hong-Wei Sun \\ shwsport@yeah.net \\ Jin-Liam Zhou \\ drzhouj1@aliyun.com \\ He-Ming Yang \\ yanghem7093@sina.com
}

Extended author information available on the last page of the article 


\section{Affiliations}

Bin-Bin $\mathrm{Li}^{1} \cdot$ Zheng-Yang Chen ${ }^{2} \cdot \mathrm{Nan}^{\mathrm{J}} \mathrm{Jiang}^{3} \cdot$ Song Guo ${ }^{2} \cdot$ Jia-Qi Yang ${ }^{2} \cdot$ Shao-Bin Chai $^{4} \cdot$ Hong-Feng Yan $^{4}$.

Pei-Ming Sun ${ }^{4} \cdot$ Gang Hu ${ }^{4} \cdot$ Tao Zhang $^{4} \cdot$ Bing-Xin Xu ${ }^{5} \cdot$ Hong-Wei Sun $^{4} \cdot$ Jin-Liam Zhou ${ }^{6} \cdot$ He-Ming Yang ${ }^{4} \cdot$ Yan Cui $^{4}$

1 Department of General Surgery, PLA 306 Clinical Hospital of Anhui Medical University, Beijing 100101, China

2 Department of General Surgery, PLA 306 Teaching Hospital of Peking University Health Science Center, Beijing 100101, China

3 Department of General Surgery, PLA 306 Teaching Hospital of Peking University Health Science Center, Beijing 100101, China
4 Department of General Surgery, PLA 306 Clinical Hospital of Anhui Medical University, Beijing 100101, China

5 Medicine and Experimental Research Center, 306 Hospital of PLA, Beijing 100101, China

6 Department of Pathology, 306 Hospital of PLA, Beijing 100101, China 